\title{
BACKWARD STOCHASTIC DIFFERENTIAL EQUATION ON HEDGING AMERICAN CONTINGENT CLAIMS
}

\author{
Ruili Song, Bo Wang \\ School of Applied Mathematics, Nanjing University of Finance and Economics, \\ Nanjing, China, 022018031@fudan.edu.cn
}

\begin{abstract}
We consider a general wealth process with a drift coefficient which is a function of the wealth process and the portfolio process with convex constraint. Existence and uniqueness of a minimal solution are established. We convert the problem of hedging American contingent claims into the problem of minimal solution of backward stochastic differential equation, and obtain the upper hedging price of American contingent claims.
\end{abstract}

Keywords- American Contingent Claim, Backward Stochastic Differential Equation

\section{INTRODUCTION}

Backward stochastic differential equations were apparently first studied in the context of the stochastic version of Pontryagin's "maximum principle" for the optimal control diffusions. These equations introduced by Pardoux and Peng [1] were useful for the theory of contingent claims valuation, especially cases with constraints. Karoui, Peng and Quenez [2] were concerned with different properties of backward stochastic differential equations and their applications to finance. Wang and Song [3] considered a more general wealth process with a drift coefficient which was Lipschitz continuous and the portfolio process with convex constraint, they converted the problem of hedging American contingent claims into the problem of minimal solution of Backward stochastic differential equation with stopping time.

In this paper, we consider a general wealth process with a drift coefficient which is a function of the wealth process and the portfolio process with convex constraint. It is shown that the problem of hedging American contingent claims can be characterized as the problem of the minimal solution of backward stochastic differential equation. Since the drift coefficient is a function of the wealth process, the backward stochastic differential equation become more complex. We adopt the penalization method for constructing the minimal solution of stochastic differential equations and obtain the upper hedging price of American contingent claims.

\section{THE MODEL}

Definition 1. (i) An F-progressively measurable process $\pi:[0, T] \times \Omega \rightarrow R^{d}$ with 


$$
\int_{0}^{T}\|\pi(t)\|^{2} d t<\infty \text { a.s. }
$$

is called portfolio process.

(ii) An F-adapted process $C:[0, T] \times \Omega \rightarrow[0,+\infty)$ with increasing, right continuous paths and $C(0)=0, C(T)<\infty$ a.s. is called cumulative consumption process.

We consider a financial market, the wealth process corresponding to a given portfolio consumption $(\pi, C)$ and initial capital $X(0)=x$ satisfies

$$
d X(t)=-f(t, X(t)) d t+\pi *(t) \sigma(t) d W(t)+d C(t)
$$

where $f:[0, T] \times \Omega \times R^{d} \rightarrow R$ is a $P \times B\left(R^{d}\right)$ progressively measurable process with $E \int_{0}^{T} f^{2}(t, \omega, 0) d t<\infty$ and

$$
\left|f\left(t, \omega, y^{\prime}\right)-f(t, \omega, y)\right| \leq k\left|y^{\prime}-y\right|
$$

$W=\left\{\left(W_{1}(t), W_{2}(t), \ldots, W_{n}(t)\right)^{*}, 0 \leq t \leq T\right\}$ is a standard d-dimensional Brownian motion and $\sigma(\cdot)=\left(\sigma_{i j}(\cdot)\right)_{1 \leq i, j \leq d}$ are bounded uniformly and progressively measurable with respect to $\mathrm{F}$.

Furthermore, $\sigma(t)$ is assumed to be invertible with $\sigma^{-1}(t)$ bounded uniformly.

\section{AMERICAN CONTINGENT CLAIMS}

Definition 2. An American contingent claim (ACC) is an F-adapted process $B:[0, T] \times \Omega \rightarrow(0,+\infty)$ with continuous sample paths and

$$
E\left[\sup _{0 \leq t \leq T}(B(t))^{2}\right]<\infty .
$$

Let us introduce now constraints on the portfolios available to agents. Suppose $K$ is a Borel subset of $R^{d}$ which contains the origin, we restrict attention to portfolios consumption rules $(\pi, C)$ that satisfy $\pi(t) \in K$.

So our class of admissible portfolio/consumption process pairs becomes now

$$
A(x)=:\left\{\left.(\pi, C)\left|\pi(t) \in K, \sup _{0 \leq t \leq T}\right| X^{x, \pi, C}\right|^{2}<\infty\right\} .
$$


Define

$$
U=:\left\{x \geq 0 \mid(\pi, C) \in A(x), X^{x, \pi, C}(\tau) \geq B(\tau), \forall \tau \in \Gamma\right\} .
$$

We introduce the upper hedging price

$$
h_{u p}(K)=: \inf \{x \geq 0 \mid x \in U\} .
$$

Let us consider the important case of convex constraints. Denote the support function of the convex set $K$ by

$$
\delta(x)=: \sup _{p \in K}\left(p^{*} x\right): R^{d} \rightarrow R \cup+\infty
$$

and its effective domain

$$
\bar{K}=:\left\{x \in R^{d} \mid \delta(x)<\infty\right\} .
$$

We shall denote by $H$ the class of F-progressively measurable processes $v:[0, T] \times \Omega \rightarrow \bar{K}$. For every $v \in H$, the exponential process

$$
Z_{v}(t)=: \exp \left\{\int_{0}^{T}\left(\sigma^{-1}(s) v(s)\right)^{*} d W^{0}(s)-\frac{1}{2} \int_{0}^{T}\left\|\sigma^{-1}(s) v(s)\right\|^{2} d s\right\}
$$

is a local martingale and a supermartingale; it is a martingale if and only if $E Z_{v}(T)=1$, in which case

$$
P^{v}(A)=: E\left[Z_{v}(T) 1_{A}\right], \quad A \in F
$$

is a probability measure and

$$
W^{v}(t)=: W(t)-\int_{0}^{t} \sigma^{-1}(s) v(s) d s
$$

is a Brownian motion. We also denote by $E^{v}$ the expectation with respect to the probability measure of $P^{v}$. In particular, this is the case for every process in the space

$$
\begin{gathered}
D=: \bigcup_{n=1}^{\infty} D_{n} \\
D_{n}=:\{v \in H \mid\|v(t, \omega)\| \leq n \text { for a.e. }(t, \omega) \in[0, T] \times \Omega\}
\end{gathered}
$$

of bounded processes in $H$.

Problem: Find a triple of F-adapted processes $(X(\cdot), \pi(\cdot), C(\cdot))$ with $(\pi, C) \in A(x)$, such that the backward stochastic differential equation(BSDE)

$$
X(t)=B(\tau)+\int_{t}^{\tau} f(s, X(s)) d s-\int_{t}^{\tau} \pi^{*}(s) \sigma(s) d W(s)+C(\tau)-C(t)
$$


and the constraint

$$
\pi(t) \in K \quad t \in[0, T]
$$

hold almost surely, and such that for any other solution $(\widetilde{X}(\cdot), \widetilde{\pi}(\cdot), \widetilde{C}(\cdot))$ satisfying (2)and (3), we have

$$
X(t) \leq \widetilde{X}(t), \quad 0 \leq t \leq T
$$

almost surely.

\section{THE UPPER HEDGING}

In order to solve the above problem, we introduce the penalized BSDE

$$
X_{n}(t)=B(\tau)+\int_{t}^{\tau}\left(f\left(s, X_{n}(s)\right)+n \rho\left(\pi_{n}(s)\right) d s-\int_{t}^{\tau} \pi_{n}^{*}(s) \sigma(s) d W(s),\right.
$$

where $\rho(y)=: \inf _{\gamma \in K}\|y-\gamma\|$ denotes the distance of the vector $y \in R^{d}$ to the set $K$. Let $X^{*}(t)=: \lim _{n \rightarrow \infty} X_{n}(t)$, then we have the following result.

Theorem 1 . The process $X^{*}(t)$ is the unique solution of the stochastic equation

$$
X^{*}(t)=\underset{v(\cdot) \in D, \tau \in \Gamma}{e s s \sup } E^{v}\left[B(\tau)+\int_{t}^{\tau}\left[f\left(s, X^{*}(s)\right)-\delta(v(s))\right] d s \mid F(t)\right]
$$

Proof: We can prove that the solution $X_{n}(t)$ of the penalized BSDE (2) satisfies the following stochastic equation

$$
X_{n}(t)=\underset{v(\cdot) \in D_{n}, \tau \in \Gamma}{\operatorname{essup}} E^{v}\left[B(\tau)+\int_{t}^{\tau}\left[f\left(s, X_{n}(s)\right)-\delta(v(s))\right] d s \mid F(t)\right]
$$

The comparison theorem for BSDE implies that

$$
X_{n}(t) \leq X_{n+1}(t), \quad 0 \leq t \leq T
$$

holds almost surely for all $n \in N$ due to $n \rho(\cdot) \leq(n+1) \rho(\cdot)$. Let $(\widetilde{X}(\cdot), \tilde{\pi}(\cdot), \widetilde{C}(\cdot))$ be any solution to the constrained BSDE, then we can prove

$$
X_{n}(t) \leq \widetilde{X}(t), \quad 0 \leq t \leq T
$$

almost surely, for every $n \in N$. We conclude that the limit 


$$
X^{*}(t)=\lim _{n \rightarrow \infty} X_{n}(t), \quad 0 \leq t \leq T
$$

exists almost surely. Fix a process $v(\cdot) \in D$ and select an integer $n$ sufficiently large, so that $v(\cdot)$ belongs to $D_{n}$, thus we have

$$
X^{*}(t) \geq X_{n}(t) \geq E^{v}\left[B(\tau)+\int_{t}^{\tau}[f(s, X(s))-\delta(v(s))] d s \mid F(t)\right]
$$

The comparison theorem implies $X_{0}(t) \leq X_{n}(t)$, for all $n \in N$, where $X_{0}(t)$ is the state process in the solution $\left(X_{0}(t), \pi_{0}(t), 0\right)$ to the unconstrained version

$$
X_{0}(t)=B(\tau)+\int_{t}^{\tau} f\left(s, X_{0}(s)\right) d s-\int_{t}^{\tau} \pi_{0}^{*}(s) \sigma(s) d W(s)
$$

of the BSDE (2). Since we also have $X_{n}(t) \leq X^{*}(t) \leq \widetilde{X}(t)$ and the Lipschitz property of $f$, we can use the dominated convergence theorem for conditional expectations to conclude that

$$
X^{*}(t) \geq E^{v}\left[B(\tau)+\int_{t}^{\tau}\left[f\left(s, X^{*}(s)\right)-\delta(v(s))\right] d s \mid F(t)\right]
$$

hold almost surely for all $v(\cdot) \in D, \tau \in \Gamma$. Thus we can get

$$
X^{*}(t) \geq \underset{v(\cdot) \in D, \tau \in \Gamma}{\operatorname{ess} \sup } E^{v}\left[B(\tau)+\int_{t}^{\tau}\left[f\left(s, X^{*}(s)\right)-\delta(v(s))\right] d s \mid F(t)\right] .
$$

The proof of the reverse inequality and the uniqueness is similar to [4].

Theorem 2. There exist processes $\left(\pi^{*}(\cdot), C^{*}(\cdot)\right)$, such that $\left(X^{*}(\cdot), \pi^{*}(\cdot), C^{*}(\cdot)\right)$ is the minimal solution to the BSDE of the above problem.

Proof: Similar to the proof of Theorem 1 in Wang [5], we have that

$$
X^{*}(t)-\int_{0}^{t}[f(s, u(s))-\delta(v(s))] d s
$$

is a supermartingale and there exist processes $\left(\pi^{*}(\cdot), C^{*}(\cdot)\right)$, such that $\left(X^{*}(\cdot), \pi^{*}(\cdot), C^{*}(\cdot)\right)$ is the minimal solution to the BSDE of the above problem.

\section{ACKNOWLEDGEMENT}

This work is supported by Science Start-up Foundation of Nanjing University of 
Finance and Economics (No.A0705) and National Youth Natural Science Foundation (No.11001119).

\section{REFERENCES}

1. E. Pardoux, S. Peng, Adopted solution of a backward stochastic differential equation. Systems, Control Lett. 14, 55-61, 1990.

2. EL Karoui, S. Peng and Quenez. M.C., Backward stochastic differential equations in finance, Math. Finance 2,1-77, 1997.

3. B. Wang and R. L. Song, The Application of Backward Stochastic Differential Equation with Stopping Time in Hedging American Contingent Claims, Chaos Solitons and Fractals 42, 2629-2634, 2009.

4. J. Cvitanic, I. Karatzas and M. Soner, Backward stochastic differential equations with constraints on the gains-process, The Annals of Probability 26, 1522-1551, 1998.

5. B. Wang and Q. X. Meng, Hedging American Contingent Claims with Constrained Portfolios under Proportional Transaction Costs, Chaos Solitons and Fractals 23, 1153-1162, 2005. 